\title{
POLIVALENCIA VERSUS ESPECIALIZACIÓN. APROXIMACIONES A UN MODELO SALUDABLE DE INTERVENCIÓN PRIMARIA
}

\section{VÍCTOR M. GIMÉNEZ BERTOMEU}

Trabajador Social. Coordinador del Centro Social Comunitario "Gastón Castelló". Servicios Sociales Municipales del Ayuntamiento de Alicante.

\section{CONCEPTOS CLAVE}

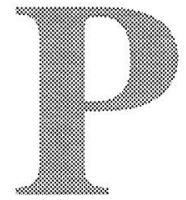

olivalencia, especialización, especificidad, intervención primaria, modelo de intervención por niveles, organización de los Servicios Sociales Generales.

\section{INTRODUCCIÓN}

Hemos oído con frecuencia a políticos, directivos y profesionales hablar de las magnificencias y ventajas de contar o ser profesionales polivalentes, que tanto valen "para un roto como para un descosido", como si de zurcir calcetines se tratara en los Servicios Sociales. Detrás de dicha concepción de lo polivalente, se esconde, en el caso del nivel político o de la alta dirección, una mal entendida polivalencia que entre líneas apunta a la economía en el número de trabajadores/as sociales y a un profundo desconocimiento del auténtico enfoque generalista que nos caracteriza como profesionales. Entre los profesionales "autocalificados" como polivalentes, traduce una peligrosa mimesis con los Servicios Sociales de Atención Primaria, que sí son polivalentes, o la ocultación de carencias en la formación y en el ejercicio.

El presente artículo pretende aportar una serie de apuntes para la reflexión acerca del ejercicio profesional de los/as trabajadores/as sociales en la organización actual de los Servicios Sociales de Atención Primaria. Con base en dos polos aparentemente opuestos, pero complementarios, la especialización y la polivalencia, se introduce un nivel intermedio de actuación, la intervención específica, que es atribuido a la Atención Primaria. 
Se apuesta por un modelo de trabajo en Atención Primaria que se fundamenta en la división del trabajo en dos niveles de intervención, el Primer Nivel (de carácter primario y más polivalente) y el Segundo Nivel (de carácter secundario y específico), y en su atribución a profesionales diferentes. Dicho sistema de intervención se ha implantado progresivamente en los Servicios Sociales Municipales de Alicante desde 1995.

\section{DEFINICIONES Y CARACTERÍSTICAS.}

\subsection{La polivalencia}

En primer lugar, se hace necesario delimitar el concepto de "polivalencia" e identificar algunas de sus características.

El Diccionario de la Lengua Española de la R.A.E.' define la polivalencia como "calidad de polivalente", es decir, que posee varios valores, y la equipara al término "plurivalente".

E. Ander-Egg, en su Diccionario del Trabajo Social, ${ }^{2}$ define lo polivalente como un adjetivo compuesto de las partículas polys "muchos" y valens "que vale", que significa que puede ser capaz de diversas cosas; que puede servir para varios usos.

Por último, Maria Sitjà, en Terminologia dels Assistens Socials ${ }^{3}$, utiliza el término "polivalente" para referirse a:

- Trabajo Social polivalente: "Trabajo social orientado a atender toda clase de problemas sociales".

- Asistente Social polivalente: "Asistente social que tiene la facultad de intervenir profesionalmente en todos los niveles del trabajo social y atender distintas clases de problemas sociales".

- Servicio Social polivalente: "Servicio social dirigido a realizar trabajo social polivalente".

De este modo, hemos encontrado fundamentalmente dos usos del término, que ya recogió Maria Sitjà:

- Con referencia a una modalidad de ejercicio profesional de los/as trabajadores/as sociales, identificándolos/as con profesionales

REAL ACADEMIA ESPAÑOLA: Diccionario de la Lengua Española (2 $1^{a}$ edición). Editorial Espasa Calpe. Madrid, 1997.

2 ANDER-EGG, E.: Diccionario del Trabajo Social. Publicaciones de la Caja de Ahorros de Alicante y Murcia. Alicante, 1981.

SITJÀ, M.: Terminologia dels Assistents Socials. Col-legi Oficial de Diplomats en Treball Social i Assistents Socials de Catalunya. Barcelona, 1988. 
polivalentes, es decir, profesionales multifuncionales que desarrollan sus competencias en servicios polivalentes. En esta última acepción, los/as trabajadores/as sociales polivalentes actúan como avanzadilla en el desarrollo de los Servicios Sociales Generales ${ }^{4}$, realizando un estudio y diagnóstico social para el desarrollo de servicios específicos y programaciones territoriales adecuados a las características de la zona.

- Con referencia a una modalidad de Servicios Sociales, los Servicios Sociales Generales, identificándolos como servicios polivalentes, es decir, servicios con la capacidad de dar respuesta a una multiplicidad de necesidades sociales. Esta acepción atribuye a los SS.SS. Generales el carácter de "primera instancia de actuación y de contacto de la población con el sistema de Servicios Sociales, de base territorial, que desarrolla funciones de información, orientación y canalización de los usuarios hacia los servicios y recursos existentes", en palabras de Josep Arenas.

Asimismo, observamos una serie de características comunes al término "polivalencia" o "polivalente" en su uso en el ámbito de la acción social:

- Implica multifuncionalidad (funciones propias y funciones bisagra).

- Atribuye un carácter primario a la intervención y al tipo de servicios.

- Supone generalidad y amplitud en el objeto de intervención.

- Significa especialización en la atención a un objeto múltiple.

\subsection{La especialización}

El Diccionario de la Lengua Española de la R.A.E. ${ }^{6}$ define la especialización como la acción o efecto de especializarse, es decir, "cultivar con especialidad una rama determinada de una ciencia o de un arte". En este sentido, señala como "especialidad" "la rama de una ciencia, arte o actividad, cuyo objeto es una parte limitada de las mismas, sobre la cual poseen saberes o habilidades muy precisos quienes la cultivan".

4 Este papel de vanguardia es recogido ya en las primeras reflexiones acerca de la organización y desarrollo de los Servicios Sociales Comunitarios. A este respecto vid. COLOMER, M. et al. Los Centros Municipales de Servicios Sociales. Documento policopiado. 1983.

5 Vid. ARENAS, J. "Seminario sobre el Contenido y Funciones de los Centros de Servicios Sociales". Revista de Servicios Sociales y Política Social n³. Consejo General de Colegios Oficiales de D.T.S. y AA.SS. Madrid, 1985. p. 22.

r REAl aCADEMia española. Op. cit. 
Por último, Maria Sitjà, en Terminologia dels Assistens Socials ${ }^{7}$, utiliza el término "especializado" para referirse a:

- Atención Especializada o Secundaria: “Atención social dirigida a la población con desventajas personales o problemas de dependencia, orientada al tratamiento, la rehabilitación y la reinserción, a la cual el usuario suele tener acceso a través de los servicios sociales de atención primaria"

- Servicio Social Especializado: "Servicio social dedicado a prestar atención especializada"

Sitjà incluye también el término "específico" con referencia al "servicio social específico", entendido como un "servicio social de apoyo a la atención primaria destinado a un sector de población y abierto a todas las personas de una zona determinada".

De este modo, como ocurría con la polivalencia, encontramos fundamentalmente dos usos del término, que también recogió Maria Sitjà:

- Con referencia a una modalidad de ejercicio profesional de los/ as trabajadores/as sociales, identificándolos/as con profesionales especializados, es decir, profesionales especialistas que desarrollan sus competencias en servicios específicos o especializados.

- Con referencia a una modalidad de Servicios Sociales, los Servicios Sociales Especializados, identificándolos como servicios especializados, es decir, servicios especialistas en dar respuesta a un sector de población o a una necesidad o grupo de necesidades. Esta acepción atribuye a los SS.SS. Especializados el carácter de segunda instancia de actuación y de contacto de la población con el sistema de Servicios Sociales, por agotamiento de las posibilidades de intervención primaria o por la idoneidad de la actuación desde un nivel especialista que desarrolle funciones de atención y rehabilitación social con medios técnicos complejos".

Podemos, por tanto, destacar una serie de características comunes al término "especialización" en su uso en el ámbito de la acción social:

- Implica funciones de alta complejidad técnica.

- Atribuye un carácter secundario a la intervención y al tipo de servicios.

- Supone limitación en el objeto de intervención.

- Significa especialización en la atención a un objeto específico. 


\subsection{La intervención primaria.}

La Intervención Primaria se lleva a cabo desde los denominados Servicios Sociales Generales, Comunitarios o de Atención Primaria. Éstos han recibido un tratamiento abundante en los trabajos profesionales e institucionales, por lo que no nos detendremos en desarrollar sus características y su contenido. En su lugar, es de interés para encuadrar el análisis que realiza este trabajo identificar brevemente las características generales que definen la Intervención Primaria":

a) Prestación técnica, que implica un nivel de cualificación profesional para su realización.

b) Básica, por cuanto es la prestación principal en la que se apoyan los SS.SS. Generales y se desarrolla desde el primer nivel de atención al ciudadano.

c) Descentralizada, al prestarse en el contexto territorial más próximo al ciudadano.

d) Dinámica, pues se desarrolla en un contexto cambiante y con el que interactúa.

e) Territorializada, al dirigirse a un ámbito geográfico de actuación determinado.

f) De carácter público, fundamentalmente.

Con variaciones en las denominaciones de las diferentes Comunidades Autónomas, la Intervención Primaria desde el Sistema Público de Servicios Sociales incluye las siguientes prestaciones básicas en respuesta a las necesidades propias del Sistema:

\begin{tabular}{|l|l|}
\hline NECESIDADES & \multicolumn{1}{|c|}{ PRESTACIONES } \\
\hline ACCESIBILIDAD & 1. Información, Orientación, Valoración y Movilización de Recursos. \\
\hline CONVIVENCIA & $\begin{array}{l}\text { 2. Apoyo a la Unidad Convivencial y Ayuda a Domicilio. } \\
\text { 3. Alojamiento alternativo. }\end{array}$ \\
\hline INTEGRACIÓN & 4. Inserción Social. \\
\hline SUBSISTENCIA & 5. Cobertura de las Necesidades de Subsistencia. \\
\hline PARTICIPACIÓN & 6. Cooperación social \\
\hline
\end{tabular}

Dado que los Servicios Sociales de Atención Primaria son competentes para la intervención territorial en dos niveles diferentes (individual/familiar y grupal/comunitario), creemos necesario reseñar que el presente trabajo se ocupa fundamentalmente del nivel individual y fa-

- Para situar adecuadamente la Intervención Primaria en el Sistema Público de Servicios Sociales, sirva como referencia el reciente "Catálogo de Prestaciones de Servicios Sociales de Atención Primaria", editado por el Ministerio de Trabajo y Asuntos Sociales. Madrid, 1998. 
miliar de la Intervención Primaria, y no del nivel grupal y comunitario de intervención, que entendemos que ha de ser objeto de una análisis diferente por cuanto aborda una realidad distinta a la individual y precisa un posicionamiento en origen también diferente. Por ello, las prestaciones y necesidades a las que nos referiremos en adelante excluirán el fomento de la solidaridad y de la participación social, más propios del nivel supraindividual.

\section{POLIVALENCIA, ESPECIALIZACIÓNE INTERVENCIÓN PRI- MARIA.}

En relación con el objetivo de este trabajo, nos interesan las acepciones de la polivalencia y la especialización que las definen como una modalidad de ejercicio profesional de los trabajadores sociales en los Servicios Sociales.

\subsection{La polivalencia y la especialización en la intervención primaria}

Como hemos podido observar, la intervención polivalente de los trabajadores sociales de los Servicios Sociales Generales consistiría en el desarrollo de:

- una prestación técnica dinámica,

- de carácter básico, primario y descentralizado,

- dirigida a un objeto múltiple: las necesidades sociales de la población general ("),

- en un territorio determinado.

Entendemos que la intervención polivalente en primera instancia es complementaria de una intervención específica que también se desarrolla desde los Servicios Sociales Generales, con la que comparte sus características principales, pero que se diferencia de la primera en:

- Su objeto de intervención, que es específico, limitado a determinadas necesidades sociales de sectores poblacionales en dificultad.

- Su carácter secundario, pues se activa tras la intervención del nivel más polivalente.

- Su contenido o prestaciones básicas que implica.

Este tipo de intervención conserva la capacidad de respuesta múltiple, característica del nivel de atención primaria, pero se hace compe-

10 Utilizamos aquí el concepto "necesidades sociales" en su sentido estricto, es decir, con referencia a las necesidades cuya cobertura es competencia del Sistema de Servicios Sociales. 
tente en dar respuesta a un grupo limitado de necesidades haciendo uso de un grupo también limitado de prestaciones. El resto de necesidades, no diagnosticadas como principales, son abordadas de forma complementaria con el resto de prestaciones de los Servicios Sociales Generales.

En consecuencia, en el continuo de la intervención social de los Servicios Sociales, distinguiríamos tres niveles profesionales interrelacionados para la intervención":

\begin{tabular}{|l|l|}
\hline \multirow{2}{*}{ INTERVENCIÓN PRIMARIA } & Intervención polivalente \\
\cline { 2 - 2 } & Intervención específica \\
\hline INTERVENCIÓN SECUNDARIA & Intervención especializada \\
\hline
\end{tabular}

De este modo, los/as trabajadores/as sociales de los Servicios Sociales Generales no son, por ello, necesariamente polivalentes en su objeto. En su defecto, pueden y deben también desarrollar intervenciones específicas que exceden al nivel más polivalente.

Como ocurre en muchas otras disciplinas, la formación académica de los/as trabajadores/as sociales prepara profesionales polivalentes que, situados en los campos de trabajo, evolucionan hacia prácticas modeladas por el objeto de intervención, el ejercicio profesional, la organización y la formación continua.

El problema surge cuando, desde los profesionales o desde los servicios, tienden a mimetizarse la polivalencia de los servicios con la polivalencia de los profesionales. $\mathrm{O}$ a atribuir a un mismo profesional funciones polivalentes y funciones específicas. Son algunas de las falsas polivalencias.

\subsection{Las falsas polivalencias y sus "ventajas".}

a) La polivalencia "forzosa".

En muchos municipios u otras entidades locales, los Servicios Sociales Generales son responsabilidad de un único profesional: el/la trabajador/a social. De este modo, el trabajador necesariamente ha de desarrollar tanto las funciones polivalentes de información, orientación y asesoramiento, como las funciones dirigidas a cubrir necesidades específicas de sectores en dificultad. Ello implica grandes dosis de soledad del profesional, de aislamiento y de elevado desgaste.

"Como hemos visto con anterioridad, estos niveles de actuación profesional fueron ya identificados en 1988 por Maria Sitjà entre los términos utilizados por los/as trabajadores/as sociales, aunque no aclara en qué nivel se sitúa la intervención específica. 
Se traduce en la infradotación de muchos Servicios Sociales, en número y tipo de profesionales, en especial en el mundo rural. El trabajador se convierte en el/la "chico/a para todo". Un/a solo/a trabajador/a social realiza desde el trabajo puramente administrativo hasta el diseño y ejecución de proyectos de intervención comunitaria, pasando por la intervención directa con individuos y familias. Muy económico.

b) La polivalencia "multiprofesional".

Se produce en contacto con otras disciplinas. Ésta es una concepción de la polivalencia que enmascara una mal entendida multidisciplinariedad o interdisciplinariedad. Significa el "todos hacemos de todo" con independencia del perfil profesional; introduce y consolida el intrusismo profesional. Supone el desaprovechamiento de lo característico de cada disciplina y la dispersión de los esfuerzos.

Surge en gran medida como mecanismo de ocultación de la propia inseguridad frente a otras profesiones, por la escasa clarificación del objeto de nuestro trabajo, nuestros objetivos, nuestro sistema característico de abordaje de la realidad o metodología y/o nuestras técnicas e instrumentos específicos de trabajo ${ }^{12}$.

c) La polivalencia "rutinaria".

Es un ejercicio típico del activismo profesional: consiste en "hacer un poco de todo y con todos", incluso tareas que no son propias del Trabajo Social. Cuando coinciden distintas disciplinas en el mismo servicio, se puede transformar en una polivalencia "multiprofesional".

Traduce el miedo a acotar el trabajo y a profundizar en un área de la realidad social. Esconde también las carencias percibidas en la formación y en el ejercicio profesional, o simplemente la inercia de una práctica que no se revisa periódica y críticamente. En otras ocasiones, es un síntoma más del agotamiento profesional.

d) La polivalencia "dogmática":

Es la polivalencia desarrollada en mimesis con la polivalencia del servicio $^{\text {i3: }}$ : El trabajador social de atención primaria es, como el ser-

12 Sirvan como ejemplo los ya habituales informes psicosociales, socioeducativos, etc. realizados ¿conjuntamente? por trabajadores sociales y otros profesionales, cuya estructura básica es la del informe social. ¿En qué medida estas prácticas contribuyen a dotar de instrumentos estandarizados de trabajo a otras profesiones a costa de perder o desvirtuar los que nos son característicos?. O en su versión más grave, los informes sociales firmados por el/la trabajador/a social y otros profesionales.

13. L. Gaitán explica este ejercicio mimético trabajador social-servicio por el papel dominante 
vicio donde se ubica, polivalente. Se localiza con más facilidad en servicios recién creados y en profesionales sin experiencia.

\section{HACIA UN MODELO SALUDABLE DE INTERVENCIÓN: LA INTERVENCIÓN POR NIVELES.}

No pretendemos recoger aquí un modelo novedoso para la intervención y la organización de los Servicios Sociales Generales. Por un lado, desde las aportaciones teóricas, ya se han propuesto modelos de organización de los Centros de Servicios Sociales a los que se asemeja nuestra propuesta ${ }^{14}$. Por otro lado, muchas entidades locales funcionan desde hace tiempo con una distribución de funciones similar. Nuestra aportación consiste en realizar aquí una lectura sintética y actualizada de dicho sistema de intervención y organización de la Intervención Primaria.

\subsection{Bases para el encuadre del modelo.}

En nuestra propuesta, el modelo de trabajo se nuclea en torno a una idea central:

Una necesidad social ha de ser atendida desde el nivel profesional más próximo al ciudadano y desde el que implique la menor complejidad técnica posible, siempre que un diagnóstico profesional no indique lo contrario.

Ello supone que, en primer lugar, se ha de utilizar un criterio económico en la intervención, bien delegando aquellas funciones que puedan ser realizadas por otros profesionales (informadores/as, auxiliares administrativos, ordenanzas, etc.), bien asignando al nivel técnico más polivalente las intervenciones menos complejas en su contenido y menos intensas en el número de actuaciones necesarias. En segundo lugar, obliga a que la propuesta de derivación del nivel más polivalente al nivel específico se realice mediante un diagnóstico de los técnicos del primer nivel.

Pero avancemos un poco en la descripción de este modelo haciendo referencia a otras de sus características definitorias:

que el trabajo social tiene en los Servicios Sociales "ya que la filosofía de éstos está inspirada en gran parte por principios de bienestar social, estrechamente asociados a los de aquella disciplina". Vid. GAITÁN, L.: "El espacio profesional", en ZAMANILLO, T. y GAITÁN,

L.: Para comprender el trabajo social. Ed. Verbo Divino. Estella, 1997. P. 104.

14 Entre otras aportaciones, véase a modo de ejemplo a GARCÍA HERRERO, G., Centros de Servicios Sociales. Conceptualización y desarrollo operativo. Ed. Siglo XXI. Madrid, 1988. 
A) Objeto.

Sin dañar el principio de universalidad que prevalece en la Intervención Primaria de los Servicios Sociales, en ésta coexisten dos objetos de intervención:

- Múltiple: las necesidades sociales de la población general.

- Específico: las necesidades sociales de sectores de la población en dificultad

B) Polivalencia versus especificidad.

Los/as trabajadores/as sociales de Intervención Primaria son competentes para el desarrollo de funciones polivalentes, dirigidas al conjunto de la población, y de funciones específicas, dirigidas a sectores poblacionales en dificultad. Y dichas funciones diferenciadas deben ser asumidas por profesionales distintos.

Es preciso destacar que el nivel polivalente, al que denominaremos Primer Nivel de Intervención, asumiría la atención primaria a la demanda, desarrollando intervenciones directas con las necesidades que les son propias, que más adelante concretaremos, o diagnosticando y derivando al nivel específico, al que denominaremos Segundo Nivel, para que éste asuma la intervención directa en el ámbito de sus competencias (necesidades-prestaciones).

C) Capacidad versus conflicto.

La intervención polivalente se ha de orientar a estimular los aspectos de capacidad que sitúan a las familias en una situación de necesidad. Por el contrario, el nivel específico de la Intervención Primaria se ha de orientar hacia los aspectos de conflicto que bloquean o inhiben esas mismas capacidades de los individuos y las familias o que sitúan en posiciones vulnerables o de riesgo a algunos de sus miembros.

Los profesionales con experiencia saben que la intervención en situaciones graves de conflicto suele ser más intensa en actuaciones y que, por lo general, se ha de incidir prioritariamente sobre los aspectos emergentes del problema que producen bloqueo o riesgo, antes de pasar a actuar sobre las capacidades de los individuos/ familias que les permitan continuar una vida normalizada. En contraposición a este tipo de situaciones, también son habituales en los Servicios de Atención Primaria las situaciones en que no existe un conflicto o una crisis grave y donde la intervención se orienta a activar potencialidades y capacidades de individuos o familias que atraviesan una coyuntura desfavorable. Por lo general, estas otras situaciones no precisan el uso de recursos técnicos que impliquen un elevado número de actuaciones. 


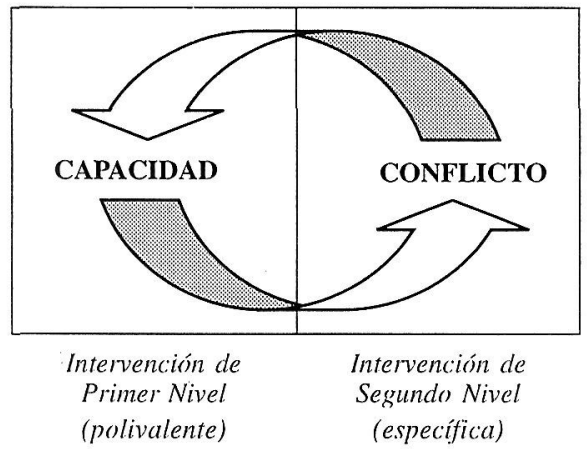

De cualquier modo, nuestra propuesta intenta no ser dogmática sino más bien flexible, y corresponderá al equipo de profesionales delimitar el grado de conflicto que puede o debe abordar el nivel más polivalente y señalar el momento en que se ha de producir una derivación interna hacia el nivel específico.

D) Intensidad.

La intensidad temporal de las intervenciones se define no tanto por la duración total de éstas como por el número de actuaciones que implica una intervención dada en un período de tiempo determinado. De este modo, el nivel polivalente desarrollará intervenciones de baja intensidad, mientras que al nivel específico le serán atribuidas las intervenciones de intensidad alta en el número de actuaciones necesarias.

E) Necesidades y prestaciones.

Como consecuencia de las características atribuidas hasta el momento a cada nivel profesional en la Intervención Primaria, se hace necesario delimitar el tipo de necesidades potenciales a que han de dar respuesta y las prestaciones cuya titularidad principal han de asumir ${ }^{15}$ :

- Nivel polivalente: Necesidades relacionadas con una adecuada información sobre el acceso a los recursos (1), con una adecuada integración social (3) y/o las relacionadas con la falta de medios de subsistencia (4). Suponen prestaciones de Información, Orientación, Valoración y Movilización de Recursos (1), de Inserción Social (4) y/o de Cobertura de las Necesidades de Subsistencia (5).

- Nivel específico: Necesidades relacionadas con una adecuada convivencia personal y familiar (2) y/o con una adecuada integración 
social (3). Supone prestaciones de Apoyo a la Unidad Convivencial y Ayuda a Domicilio (2), de Alojamiento alternativo (3) y/o de Inserción Social (4). Además, desde una perspectiva global de la intervención, utiliza el resto de prestaciones con carácter complementario.

3.2. Características del modelo: una combinatoria de factores.

\begin{tabular}{|l|l|l|}
\cline { 2 - 3 } \multicolumn{1}{c|}{} & $\begin{array}{l}\text { INTERVENCIÓN DE } \\
\text { PRIMER NIVEL }\end{array}$ & $\begin{array}{l}\text { INTERVENCION DE } \\
\text { SEGUNDO NIVEL }\end{array}$ \\
\hline OB.JETO & $\begin{array}{l}\text { Múltiple (Necesidades de la } \\
\text { población en general) }\end{array}$ & $\begin{array}{l}\text { Específico (Necesidades de } \\
\text { sectores en dificultad) }\end{array}$ \\
\hline PRÁCTICA & Polivalente & Específica \\
\hline ORIENTACIÓN & Capacidad & Conflicto \\
\hline INTENSIDAD & Baja & Alta \\
\hline NECESIDADES ${ }^{16}$ & $1,3,4$ & 2,3 \\
\hline PRESTACIONES ${ }^{17}$ & $1,4,5$ & $2,3,4$ \\
\hline
\end{tabular}

\begin{tabular}{|l|l|l|}
\hline EQUIPO & "Uniprofesional" & Multiprofesional \\
\hline METODOLOGÍA & Trabajo Social & $\begin{array}{l}\text { Multidisciplinar } \\
\text { Interdisciplinar }\end{array}$ \\
\hline ALCANCE & Alto & Limitado \\
\hline
\end{tabular}

Además de las características que ya señalamos, este modelo de división del trabajo en la atención primaria implica que:

- El nivel polivalente sea asumido por trabajadores/as sociales, junto a personal auxiliar, mientras que el nivel específico ha de tender a contar con profesionales de diferentes disciplinas (Trabajo Social, Psicología, Educación Social, etc.).

- La metodología de intervención en el nivel polivalente sea básicamente la propia del Trabajo Social, mientras que la metodología del segundo nivel ha de tender a la multidisciplinariedad, en un primer grado, y a la interdisciplinariedad, en un segundo grado.

- El número de situaciones abordables desde el primer nivel sea más elevado que en el caso del nivel específico, cuyo alcance es más limitado.

16. Remitimos al Manual de Cumplimentación de la Ficha Social editado por el Ministerio de Asuntos Sociales y las Comunidades Autónomas.

17 Remitimos al Manual de Cumplimentación de la Ficha Social editado por el Ministerio de Asuntos Sociales y las Comunidades Autónomas. 
Apostamos también en el modelo por que la intervención indirecta (la responsabilidad de la gestión y seguimiento de los programas de prestaciones básicas) sea competencia del director/a o coordinador/a del Centro de Servicios Sociales con el apoyo que proceda, mientras que la aplicación y seguimiento singular de las prestaciones corresponda a los profesionales de atención directa de cualquiera de los niveles. Ello en contraposición a la distribución de la responsabilidad sobre los programas entre profesionales de uno u otro nivel, tarea que entonces habrían de compatibilizar con la intervención directa. Entendemos que asumir parte de la gestión y seguimiento de los programas acaba limitando a los profesionales de uno y otro nivel al orientar su trabajo a los recursos disponibles y no ampliarlo a los recursos idóneos. A riesgo de caer en la perogrullada, corresponde al director/a promover los recursos suficientes y necesarios que los profesionales precisan para su trabajo. O dicho en otras palabras, los profesionales han de determinar las herramientas que necesitan y los/as directores/as han de intentar facilitárselas.

\subsection{Condiciones mínimas para la posibilidad del modelo.}

A) Concepción del trabajador social de Atención Primaria.

Adoptar un modelo de trabajo de estas características supone concebir al trabajador/a social en Intervención Primaria tal y como lo hemos definido a lo largo del artículo:

\begin{tabular}{|l|c|c|}
\cline { 2 - 3 } \multicolumn{1}{c|}{} & $\begin{array}{c}\text { INTERVENCIÓNDE } \\
\text { PRIMER NIVEL }\end{array}$ & $\begin{array}{c}\text { INTERVENCIÓNDE } \\
\text { SEGUNDO NIVEL }\end{array}$ \\
\hline FORMACIÓN Académica & \multicolumn{2}{c|}{ Polivalente y multidisciplinar } \\
\hline FORMACIÓN Continua & Enfoque generalista adecuado al objeto/sujeto de intervención \\
\hline PRÁCTICA & Múltiple & Específico \\
\hline OBJETO & \multicolumn{2}{c}{} \\
\hline
\end{tabular}

B) Enfoque generalista.

Situarnos en este modelo de trabajo no significa abandonar el enfoque generalista en ninguno de los niveles de actuación señalados. Como recoge la National Asociation of Social Workers americana (N.A.S.W.) en su Enciclopedia de Trabajo Social"s, "la prácti-

Is Acerca de la perspectiva generalista del Trabajo Social, la literatura profesional estadounidense ha producido un interesante artículo de B.D. SHEAFOR y P.S. LANDON "Generalist Perspective". en Encyclopedia of Social Work (18"t edition). National Asociation of Social Workers. Silver Spring, MD (U.S.A.), 1987. Pp. 660-669 (Volumen I). La cita es de la página 664 . 
ca generalista implica tanto la capacidad de tener una visión global de la situación concreta como las habilidades necesarias para intervenir en niveles múltiples y en una variedad de situaciones".

Entendemos, con L. Gaitán, que este enfoque, elemento característico y definitorio de los/as trabajadores/as sociales frente a otras profesiones, es especialmente útil en la atención social primaria "por partir de una premisa de globalización (no parcelación o compartimentación) de los problemas, por su capacidad discriminante acerca del tipo, modo, duración y lugar más adecuado para la atención de los problemas, a partir de la valoración (global) de la situación planteada, y por su flexibilidad y adaptabilidad a las características concretas de la/s persona/s, el entorno y el problema"!".

C) Profesionales.

Como se puede deducir por lo expuesto hasta el momento, implica también contar con un número suficiente de trabajadores/as sociales (como mínimo, dos) a situar en cada nivel de intervención.

Además, supone contar con otros profesionales (psicólogos/as, educadores/as, trabajadores/as, familiares, etc.) que se integren en un equipo multiprofesional.

D) Trabajo en equipo.

Además no basta con que contemos con suficientes y variados profesionales, sino que éstos han de actuar como un verdadero equipo, sirviendo éste como instancia de consulta, estudio y supervisión del trabajo de cada nivel de intervención.

E) División del trabajo.

El modelo se traduce en una división del trabajo entre el nivel directivo y el nivel operativo y, dentro de este último, en una diferenciación funcional según el objeto de la intervención.

F) Formación.

Los profesionales han de estar formados adecuadamente para trabajar con el objeto/sujeto de su intervención. Y en este punto nos parece especialmente importante destacar que tan formados han de estar los profesionales del segundo nivel, cuyo objeto es más limitado, como los/as trabajadores/as sociales polivalentes, ya que estos últimos, entre otras funciones, son auténticos especialistas en intervenciones urgentes y breves ${ }^{20} \mathrm{y}$ en la realización a corto plazo

19) En cuanto al enfoque generalista de los/as trabajadores/as sociales de atención primaria en España, me parece especialmente relevante la lectura de GAITÁN, L. "El enfoque generalista del trabajo social", en Revista de Treball Social n ${ }^{\circ}$ 150. Col-legi Oficial de D.T.S. i AA.SS. de Catalunya. Barcelona, 1998. Pp. 6-42. La cita que incluimos es de la página 15.

20. Vid. DE ALFONSETI, N. "La figura del Trabajador Social en Servicios Generales del Sector 
de diagnósticos de paso al nivel específico de intervención.

G) Equipamientos.

Si hablamos de profesionales diferenciados funcionalmente, consecuentemente hemos de hacer referencia a la necesidad de que éstos cuenten también con espacios diferenciados dentro de los Centros de Servicios Sociales. Este aspecto no habría que mencionarlo si realmente existiera una red de Centros de Servicios Sociales adecuada y suficiente, con "auténticos" despachos para los profesionales, en lugar de sótanos y locales sin ventilación o pisos inaccesibles, por citar algunos penosos ejemplos. En nuestro planteamiento, el contexto físico donde el trabajo se lleva a cabo es fundamental para el buen ejercicio de los profesionales y para la digna atención que merece el ciudadano.

\subsection{Aspectos "saludables" del modelo.}

Para finalizar, cabe reseñar algunos de los elementos que al parecer del autor hacen "saludable" este modelo de organización de la atención primaria de los Servicios Sociales, tanto para los profesionales como para los destinatarios finales del mismo:

A) La división del trabajo.

El "todos hacemos de todo" se sustituye por el "cada uno hace una parte del todo", entendiendo por el "todo" el conjunto de áreas de necesidad en las que interviene el Centro de Servicios Sociales. No consiste en que una brigada de trabajadores sociales, especialistas cada uno en un área de necesidad, desembarque en la realidad individual o familiar para repararla, sino que un único/a trabajador/ a social asuma el papel aglutinador de la intervención y de los demás profesionales, y haga uso con ellos de todos los instrumentos y recursos disponibles y necesarios de los que dispone el Servicio.

B) La concreción del objeto de trabajo.

Se ha hablado de la dispersión de los esfuerzos de los profesionales de la Atención Primaria al tener que abordar una gran variedad de situaciones sociales. Este sistema permite a los profesionales acotar su objeto de trabajo a unas áreas de necesidad cuyo abordaje es competencia de los Servicios Sociales Municipales.

C) La asignación de casos.

Este sistema permite que las situaciones familiares más críticas, en las que actúa el Segundo Nivel, sean seguidas por el mismo tra-

Público", en DÍ. Aportes desde el Trabajo Social n 7/8. Colegio Oficial de D.T.S. y AA. SS. de Alicante. 1998. P. 16. 
bajador/a social, hasta que un diagnóstico profesional determine el cierre de la intervención o derive al Primer Nivel.

Compartimos con V. Coulshed ${ }^{21}$ que se trata de un método que no sólo afecta a la organización del tiempo, sino también a la calidad del servicio ofrecido al ciudadano al asignar la continuidad de la intervención en una situación problema a un único trabajador/a social y no a cualquier trabajador/a social del Servicio. Incide también en la productividad y en el volumen de casos tratados. Como método que deriva de la gestión por objetivos, se fundamenta en la valoración continua y en la revisión de la intervención.

Este sistema de organización interna de los servicios, se contrapone a lo que Vickery ${ }^{22}$ denominó «círculo de la práctica caótica» como definición de un trabajo no planificado y reactivo en el que los/as trabajadores/as sociales sólo responden a las crisis en lugar de desarrollar una intervención programada. De modo que la intervención se orienta a los «problemas visibles», con lo que se da una prioridad mínima a la valoración y a la revisión, favoreciendo que muchas situaciones se transformen en «crónicas».

D) La práctica específica.

Como consecuencia directa de la limitación del objeto de intervención, la práctica profesional se hace específica y más concreta en respuesta a unas necesidades limitadas.

E) La formación específica.

Un objeto y una práctica concretados en determinadas áreas de necesidad permiten orientar de manera más concreta la formación continua del trabajador/a, para dotarlo/a de habilidades y técnicas específicas de intervención adaptadas al objeto/sujeto de la actuación profesional.

F) La distribución de la carga de trabajo.

Aunque existen también otros mecanismos para equilibrar el volumen de trabajo de los diferentes trabajadores/as sociales, con este modelo de trabajo, el/la responsable del equipo de intervención, junto a los/as profesionales, puede situar con flexibilidad los límites y las áreas de necesidad en que actuarán uno u otro nivel en función de la carga de trabajo que representan para los profesionales.

21 COULSHED, V. La gestión del trabajo social. Paidós. Barcelona, 1998 (Edición original inglesa de 1990). P. 104.

22 VICKERY, A. Caseload Managenent. National Institut for Social Work. Londres, 1977. Citado por COULSHED, V. La gestión del trabajo social. Paidós. Barcelona, 1998 (Edición original inglesa de 1990). Pp. 105-107. 


\section{G) La rotación.}

Estamos convencidos que la intervención primaria provoca elevadas dosis de desgaste en los profesionales sometidos al mismo tipo de intervención y a actuar con las mismas necesidades y, muchas veces, con la misma población durante años. Además, la promoción interna de los/as trabajadores/as sociales en los Servicios Sociales Municipales está normalmente limitada al acceso a puestos de gestión de Programas o de Centros de Servicios Sociales, si un titulado superior no lo ha hecho ya.

Con este sistema de abordaje de la realidad local, los profesionales pueden establecer un sistema de rotación entre niveles, que les permita ampliar mínimamente sus posibilidades de alternancia y cambio en las tareas de atención directa.

De la experiencia vivida en la aplicación de este modelo, creemos que el período mínimo de permanencia en cada nivel ha de ser de 2 años. El intervalo concreto vendrá condicionado por la organización general del servicio, por los aspectos relacionados con la situación laboral de los trabajadores y por los ritmos de las familias, que aconsejen adelantar o retrasar la derivación de la intervención a otro profesional.

H) La formación y la perspectiva integrales.

La rotación permite el paso de los/as trabajadores sociales del Centro por los diferentes niveles de intervención, y en consecuencia se amplía el foco necesario para el diagnóstico global de las situaciones y se refuerza la perspectiva generalista. Además enriquece la formación integral del profesional. 